\title{
Professional v/s Personal: How to Make it Work
}

\author{
Hannah Elizabeth Mathew ${ }^{1}$, Akshaya Ramachandran², Kavinya Karunakaran ${ }^{3}$, Sohag \\ Bagchi $^{4}$, Vignesh Mansingh ${ }^{5}$ \\ 1,2,3,4,5 Semester VIII Medical Students, Kasturba Medical College, Manipal University, Manipal
}

\begin{abstract}
This writing deliberates upon how to achieve an optimal work-life balance, considering the professional and personal spheres of an individual's life. With a detailed step to step explanation of how one can achieve work-life balance, the article concludes by emphasizing upon the individual differences that one must always consider. Work life balance is an important establishment in every individual's life as it allows one to live optimally at a personal and professional level. Nevertheless, the bigger picture in everyone's life is different and has its one own relevance to the individual.
\end{abstract}

Key Words: professional life, personal life, work-life balance

We as physicians - present and future - all begin our education and careers striving to save the world by healing one ill patient at a time. Our drive, dedication and devotion towards restoring the sick to health push us into long days and nights studying and practicing to attain our goal. In this process of medical school we tend to get caught up in all of our textbooks, practical and cases; however, we look past these difficult times towards the light at the end of the tunnel, which is seeing our patients hale and healthy. Naively we believe that life becomes easier once our education has been completed only to realize that clinical practice involves a whole new set of hurdles that we must overcome. Not only do these hurdles include our professional workload, but also our personal lives which continue to grow and move forward as we do.

Our attempt to balance professional and personal lives may not go as we intend: this puts us at a great risk of developing the dreaded burnout. This burnout causes us to become disgruntled, which can then affect our patients negatively (physically and emotionally, among other aspects of their health), causing them to exhibit noncompliance or even worsen their condition. If the patient continues to deteriorate, our work load and stress level rises in a futile attempt to reestablish the patient to their previous healthy state thus creating a vicious cycle. In order to prevent this vicious cycle in taking over our commitment to the field of medicine and the patients we serve, we must learn to balance our professional and personal life so that we may be able to treat our patients with the utmost care while also taking into account our personal lifestyle (health, family, pleasure and leisure).

Before we delve into how to achieve proper work life balance, we must first understand what it entails. Work consists of our ambitions, goals and careers. Life contains not only our friends and 
family, but also the way we see and believe in ourselves. Work life balance is "satisfaction with one's entire life-professional and personal." This does not necessarily mean that both should have a fifty-fifty allocation of our time, energy and resources. However, we must keep in mind that each person has their own definition of balance-some may perform better with longer working days while others may prefer more time with their loved ones in order to recharge and be better suited to take on the day's work [1].

Now that we have an understanding of work life balance, we must also know the consequences of its absence. These consequences include, but are not limited to, poor health, exhaustion and strain on our relationships. Long days with little sleep cause us to suffer from exhaustion, which may obscure our ability to treat our patients effectively. This will not only cause damage to the patient's wellbeing but also our professional reputation. Furthermore, with increased pressure at work, we are more prone to develop stress. Stress is a known causative factor for several medical conditions including weakened immune system, heart related disorders, mental disorders and the list goes on. Additionally, stress puts us at a higher risk for substance abuse and suicide. Moreover, if we work excessively, we will miss important moments with our friends and family. This can lead to an inability to build and nurture relationships with others which can lead to isolation and the negative psychosocial effects that it may bring [2].

One of the primary steps in achieving a work life balance is learning how to prioritize our careers with our lifestyles. We must spend time truly trying to figure out what we hold most dear and remove the unnecessary things that eat away at our precious time. Everyone will have different priorities, so it is essential to prioritize based on our own needs rather than those of others. We must also remain firm in the decisions we have made regarding these priorities in order to reap the benefits. To prioritize our needs and desires, we must realize that compromise is a necessity. By learning how to compromise, we begin to understand what we truly hold dear, even though it may mean not spending as much time as we previously thought we would in the hospital with our patients [1].

The next step becomes increasingly challenging in this day and age: we must learn how to put down our phones and unplug once in a while. In this day and age, technology has become one of the greatest assets in our professional as well as personal lives. We are able to communicate with patients and diagnose conditions like never before. However, technology comes with a cost. This constant connection to work inhibits us from truly leaving and dedicating our energy elsewhere. Patients may call after hours, colleagues may want a second opinion, insurance companies may seek to clarify claims, etc. In doing so, we end up stepping away from our other endeavors just to be drawn back into work. Hence, we must exercise control over separating work from play. It is required that we make quality time truly count by disconnecting from work when we are with our loved ones [3].

Moreover, we must practice what we preach when it comes to exercise and relaxation. It is necessary to take a few minutes for ourselves to unwind and rejuvenate [3]. This in turn has been shown to increase productivity and decreases inefficiency.

Finally, none of this will happen overnight. It is crucial to begin small and gradually progress to achieve success [3]. We need to take baby steps, one at a time, before we can appreciate the results. It is also important not to get discouraged when we fumble. 
Therefore, to put everything we have discussed so far into perspective we need to step back and look at the big picture. Every person will have a different picture staring back at them, but in an effort to achieve a healthy balance we need to follow these four simple but essential steps: prioritize, compromise, exercise, and start small. With these in mind, our initial spark and drive to heal our patients can stay aflame despite our realization that we are also merely humans who have their own necessities that need to be addressed from time to time.

\section{REFERENCES}

1. Berry E. Achieving work-life balance: more than just a juggling act. http://www.amednews.com/article/20100104/business/301049968/4/ (accessed 17 March 2016).

2. Mayo Clinic Staff. Work-life balance: tips to reclaim control. http://www.mayoclinic.org/healthylifestyle/adult-health/in-depth/work-life-balance/art-20048134 (accessed 17 March2016).

3. Lee DJ. 6 Tips for better work-life balance. http://www.forbes.com/sites/deborahlee/2014/10/20/6tips-for-better-work-life-balance/\#3d78b163dbc9 (accessed 17 March 2016).

$* * * * * * * * * * * * * * * * * * * * * * * * * * * * * * * * *$

Acknowledgments - Nil

Source of Funding - Nil

Conflict of Interest $-\mathrm{Nil}$ 